\title{
Validación de una escala de autoeficacia para la prevención del SIDA en adolescentes
}

Fuensanta López-Rosales, Dra. en Psic. ${ }^{(1)}$ José Moral-de la Rubia, D r. en Psic.(2)

\section{López-Rosales F, Moral-de la Rubia J. Validación de una escala de autoeficacia para la prevención del SIDA en adolescentes. Salud Publica Mex 2001;43:421-432.}

El texto completo en inglés de este artículo está disponible en: http://www.insp.mx/salud/index.html

\section{Resumen}

Objetivo. Estudiar la consistencia interna, validez y estructura factorial de la escala Self-Efficacy for AIDS (SEA-27) desarrollada en población estadounidense y adaptada a población mexicana. Material y métodos. Es un estudio correlacional, con un diseño no experimental de tipo transversal. Se hizo con una muestra aleatoria simple de 964 adolescentes tomados de 58 centros de salud públicos de Monterrey, México, en febrero de 1998. N inguno iba a consultar por motivos relacionados con el SIDA. Se emplea la Escala deA Autoeficacia de 27 elementos para Prevenir el SIDA (SEA-27) de López Rosales y colaboradores, junto con preguntas cerradas tipo Likert, dicotómicas y un cuestionario integrado por dos preguntas abiertas sobre aspectos relevantes de la conducta sexual para validar la escala. Para el estudio de SEA 27 se emplearon las pruebas $U$ de Mann W hitney, Kolmo gorov-Smirnov, alpha de C ronbach, análisis factorial de componentes principales y correlación bi-serial puntual. La validez de la escala se estudia por la correlación de Pearson con las variables numéricas, con la t de Student para muestras independientes dicotómicas y AN OVA con las politómicas. Resultados. La escala se ajustó a una distribución normal $\left(Z_{k_{\text {- } s}(\mathrm{~N} . \text { ormall }} 1.369 ; p_{\Sigma} 0.047\right)$, con una media de 95.14 y una desviacion estándar de 25.80. Su fiabilidad, por la alpha de Cronbach, resultó de 0.89. SEA-27 muestra una estructura tetradimensional ortogonal que explica $58.47 \%$ de la varianza. Se confirmaron en buen grado las

\author{
López-Rosales F, Moral-de la Rubia J. \\ Validation of a self-efficacy scale \\ for AIDS prevention among adolescents. \\ Salud Publica Mex 2001;43:421-432. \\ The English version of this paper \\ is available at: http://www.insp.mx/salud/index.html
}

\begin{abstract}
A bstract
Objectives The objectives are obtain the internal consistency, validity and factorial structure of the Self-Efficacy for AIDS scale, wich was developed in A merican population by Kasen,Vaughan and W alter (1992) and adapted in mexican samples for López and Padilla (1999). Instruments: It is employed the Self-efficacy to Prevent theAIDS Scale of 27 items of López and Padilla (1999), together with closed questions Likert type, dicotomics and two opened questions on relevant aspects of the sexual conduct to validate the scale, under a format self-applied test of pencil and paper. Material and Methods it is a correlacional study with a not experimental design of transverse type. It was accomplished with a simple random sample of 962 adolescents wich were taken of 60 public health centers of Monterrey. $\mathrm{N}$ one went to consult by motives related to the AIDS. Results. The scale was adjusted to a normal distribution (Zk-s(N ormal) $=1.369 ; p=0.047)$. It presents a mean of 95.14 and a standard deviation of 25.80 . Its reliability, by the alpha of Cronbach, was 0.89 . SEA - 27 shows a structure of four independent factors that explain $58.47 \%$ of the total variance. Conclusions Thus, it is concluded that SEA - 27 is a reliable and valid scale.The English version of this paper is available at: http://www.insp.mx/salud/index.html
\end{abstract}

(1) Centro de Investigaciones de la Facultad de Psicología de la Universidad Autónoma de N uevo León, México.

(2) Universidad Autónoma de N uevo León, México.

Fecha de recibido: 26 de octubre de 2000 - Fecha de aprobado: 4 de junio de 2001 Solicitud de sobretiros: D ra. Fuensanta López Rosales. Centro de Investigaciones. Facultad de Psicología. Universidad Autónoma de N uevo León. C/Mutualismo 110. Colonia Mitras Centro. 64460. Monterrey, N uevo León, México. Correo electrónico:flopez@ccr.dsi.uanl.mx,jose_moral@ hotmail.com 
hipótesis de validez correlacional y discriminatoria. Conclusiones La SEA-27 es una escala fiable y válida. El texto completo en inglés de este artículo esta disponible en: http://www.insp.mx/salud/index.html

Palabras claves: auto eficacia; síndrome de immunodeficiencia adquirida; adolescencia; conducta sexual de riesgo; psicometría; México
Key words: self-efficacy; acquired immunodeficiency syndrome; adolescence; sexual behaviour of risk; psychometrics; Mexico
E 1 presente artículo aborda el problema de la prevención del contagio por VIH en adolescentes mexicanos, en un momento en el cual la epidemia registra una tasa incidencia creciente frente a una clara disminución de nuevos casos en los países occidentales más desarrollados (ONUAIDS, 2001). ${ }^{1}$

En el año 2000, según la Dirección General de Epidemiología (DGE) del Consejo Nacional de Prevención y Control de SIDA (CONASIDA), hay registrados 867 casos de SIDA en el estado de Nuevo León, lo que representa 2.95\% de los 29419 casos del total nacional, siendo $4 \%$ de los infectados menores de 15 años, $72 \%$ tendría una edad comprendida entre 15 y 44 años y $24 \%$, más de 44 años. ${ }^{2}$ La probabilidad de hallar en México un caso de SIDA diagnosticado en las edades comprendidas entre los 11 y 19 años sería aproximadamente de 1 cada 10000 adolescentes.

En este estudio enfocamos la prevención desde la autoeficacia para adquirir y mantener conductas que eviten el contagio por VIH. El concepto de autoeficacia se basa en la teoría sociocognitiva de Bandura. ${ }^{3}$ Con autoeficacia nos referimos a la percepción por parte del adolescente de su competencia para desarrollar una conducta sexual eficaz para prevenir el SIDA. Según Bandura, ${ }^{4}$ los cuatro factores que incrementan la autoeficacia son las experiencias de éxito, el éxito vicario, la persuasión social y la reducción de la ansiedad, siendo un requisito la atribución interna del control y éxito de la conducta.

Para medir la autoeficacia percibida en el mantenimiento de una conducta sexual preventiva del SIDA, Kasen, Vaughan y Walter ${ }^{5}$ crearon una escala de 22 reactivos. Su estudio se desarrolló en una muestra de adolescentes, hombres y mujeres, de 15 a 19 años, en la ciudad de Nueva York. Se reportó un nivel de consistencia interna alto $(\alpha=0.91)$ y de validez con base en su capacidad discriminatoria de conductas de riesgo.

Esta escala ha sido adaptada al español y estudiada en muestras mexicanas. ${ }^{6,7}$ Ambos estudios reportan un nivel de consistencia interna alto $(\alpha=0.91)$ y una buena validez discriminatoria. Kasen y colaboradores ${ }^{5}$ encon- traron un nivel alto de autoeficacia en la utilización del preservativo, no así en referencia al rechazo de relaciones sexuales; Bayés y colaboradores ${ }^{6}$ hallaron que en su población hay mayor autoeficacia en el rechazo de relaciones sexuales, y menor en el uso de preservativos. En ambos estudios los hombres se perciben más autoeficaces para usar el preservativo y las mujeres para rechazar relaciones sexuales.

López Rosales y colaboradores ${ }^{7-9}$ aplicaron la SelfEfficacy for AIDS en una muestra de 3265 estudiantes neoleoneses de secundaria. No obstante, efectuaron algunas modificaciones en la escala, pasando ésta de 22 a 27 elementos. Dichos cambios fueron motivados por la dificultad hallada para entender algunos ítem o ambigüedad en la respuesta. A esta versión de 27 elementos la vamos a denominar SEA-27. Se volvió a hallar un nivel de consistencia interna alto de 0.91, calculado por la alpha de Cronbach. Por otra parte, se reporta un mayor nivel de autoeficacia en las clases sociales más altas y un bajo uso de preservativo en la muestra general ( $43 \%$ nunca lo usa y $18.4 \%$, a veces). El $15.83 \%$ de los varones habían tenido relaciones sexuales y $5.11 \%$ de las mujeres. La edad modal para el inicio de las relaciones sexuales son los 14 años en los varones y 16 en las mujeres.

Los objetivos del presente trabajo son calcular la confiabilidad y validez de la Self-Efficacy for AIDS (SEA-27), tanto de sus 27 elementos como de la suma total de elementos y su estructura factorial. Asimismo, estudiar la validez de constructo, a partir de la estructura factorial subyacente y desde un enfoque correlacional y discriminatorio, empleando un conjunto de variables referidas a la actitud hacia el uso de preservativo, la planificación de la relación sexual y la monogamia, información que se posee en relación con el contagio de VIH y conducta sexual que se mantiene.

\section{Material y métodos}

El estudio se realizó en una muestra de 964 adolescentes tomados de 58 centros de salud públicos de Monterrey y sus municipios colindantes (San Pedro 
Garza García, San Nicolás de los Garza, Guadalupe, Santa Catarina, Escobedo y Apodaca). La muestra fue capturada en febrero de 1998.

La edad media es de 16.24 años con una desviación estándar de 2.09, una mediana de 16 y una moda de 15 años. La edad mínima fue 12 y la máxima 27. La distribución de edad describe una curva leptocúrtica, concentrándose 70\% de los casos entre los 14 y 17 años, pues precisamente se trata de una muestra de adolescentes. No se consideró el motivo de consulta para la selección de la muestra. Ningún sujeto tenía diagnóstico de SIDA y el porcentaje de $\mathrm{VIH}+$ es desconocido. El número medio de personas que conviven en la casa del adolescente son $5 \pm 2.4$, en $74 \%$ de los casos el papá, la mamá y los hermanos. En $26 \%$ hay otros parientes viviendo en la casa (abuelos o primos).

En cuanto al estado civil, $85.1 \%$ de los sujetos son solteros, $11.7 \%$ casados, $2.7 \%$ separados o divorciados y $0.5 \%$ son parejas en unión libre. El $61.8 \%$ pertenecen a la clase social media-baja, $36.3 \%$ a la clase baja, $1.5 \%$ a la media-alta y $0.4 \%$ a la clase alta. El nivel de estudio modal es de secundaria $(71.6 \%)$, seguido de preparatoria $(13.5 \%)$, primaria $(13.3 \%)$ y universidad $(1.6 \%)$.

El 57\% son mujeres y $43 \%$ varones. El 70\% estudia y $30 \%$ ya ha abandonado los estudios. El 28\% trabaja y $72 \%$ dice no trabajar. El 30\% de los encuestados afirman haber mantenido relaciones sexuales, $34 \%$ de los varones y $27 \%$ de las mujeres.

Se recogieron datos de variables sociodemográficas y socioeconómicas y la autoeficacia se mide a través del Self-Efficacy for AIDS, ${ }^{3}$ tomando su formato del estudio de López Rosales y colaboradores ${ }^{7}$ (SEA27). Dicho instrumento de medida consta de 27 reactivos tipo Likert con una variación de cinco puntos (Apéndice), evalúa la autoeficacia desde la teoría sociocognitiva de Bandura, ${ }^{4}$ en tres aspectos de la conducta relacionada con el sexo:

- Capacidad para decir no a las relaciones sexuales bajo diferentes circunstancias.

- Capacidad percibida para preguntar al compañero sobre las relaciones sexuales anteriores y otras conductas de riesgo como consumo de drogas.

- Capacidad percibida para adquirir y utilizar correctamente preservativos.

La suma de estos 27 elementos constituye la variable ST como una puntuación total de autoeficacia percibida para prevenir el SIDA con relación a conductas sexuales de riesgo, que en este trabajo, a veces, también aparece bajo la denominación de escala SEA27. Al ajustarse a una distribución normal, una vez estandarizada con base en su media y desviación es- tándar, se puede considerar una variable numérica en una escala de intervalo.

Se mide la actitud hacia el uso de los preservativos y planificación sexual con base en 10 variables de orden. Están construidas como reactivos tipo Likert con una variación de cinco puntos. Además, hay una pregunta sobre la actitud hacia la monogamia.

A su vez, como variable dicotómica se mide el haber tenido o no relaciones sexuales. En caso de contestación afirmativa, se evalúan como variable dicotómica si se utilizó o no presevativo en la primera relación con coito, el haberla o no planificado, el cómo y dónde de la relación sexual y si se emplea el preservativo en las relaciones actuales. Como variables cuantitativas se pide el número de personas con las que se ha tenido relaciones sexuales y la edad a la que se tuvo esa primera relación sexual.

En el supuesto de haberse tenido al menos una relación sexual, y no haber usado preservativo, se evalúa como variable politómica la razón por la que no se empleó éste. Por otra parte, se solicita a los sujetos que estimen la efectividad del preservativo, en una variable cualitativa politómica con tres categorías: a) 95 a $100 \%$, b) 90 a $100 \%$ y c) menos de $90 \%$. Como variable de orden (1 a 5), se pregunta al sujeto si está conforme con que es muy difícil que se infecte de SIDA, aunque no se proteja.

También se pregunta sobre la información que poseen acerca de la sexualidad, de donde se extraen tres variables. Una numérica de orden donde el sujeto evalúa en qué grado es buena o mala la información que posee ( 1 al 5); y las otras dos politómicas: sobre qué tema le gustaría tener más información, y la fuente principal de su información (amigos, libros, revistas, periódicos, televisión, escuela, papá o mamá).

Finalmente, especificamos 15 conductas de riesgo y se pide al sujeto que escoja tres y las ordene según su importancia $\left(1^{\circ}, 2^{\circ}\right.$ y $\left.3^{\circ}\right)$ como factor de riesgo para infectarse con el VIH. Con estos últimos datos definimos 15 variables ordinales. Cada una de las 15 opciones $(A, B, \ldots ., O)$ define una variable ordinal (riesgo $A$, ries$g_{0} B, \ldots .$, riesgo $\left.O\right)$ con un recorrido de 1 a 3 , indicando importancia del riesgo atribuido o lugar de elección (1 en primera opción, 2 en segunda opción y 3 en tercera opción) (apéndice).

Todas estas variables especificadas permiten estudiar la validez de la escala Self-Efficacy for AIDS (SEA-27) y se obtuvieron mediante una encuesta autoadministrada (apéndice) la cual tarda entre 40 y 50 minutos en completarse. Fue aplicada por personal del Centro de Salud, después de que los investigadores pertenecientes al proyecto explicaran la finalidad del estudio y la forma de administración del cuestionario, 
motivaran al personal para su aplicación y se facilitaran los ejemplares. De todo el personal del centro, los promotores sociales fueron quienes más colaboraron y quienes realmente realizaron el proceso de muestreo no probabilístico.

Los centros de salud se eligieron al azar entre el total de ellos en los municipios en estudio (58/240); los pacientes se tomaron del total de consultas practicadas en un día no festivo (de 15 a 20 personas por centro, con una media de 78 consultas de medicina general por día; por lo tanto se trata de una muestra aleatoria simple.

Para los cálculos estadísticos se empleó el SPSS8.0, aunque los datos fueron capturados con la hoja de cálculo Excel 97.

Se determinó el tipo de distribución y estadísticos descriptivos de la escala definida como suma total de elementos (ST); se contrastó el ajuste de la distribución a una población normal con la prueba de KolmogorovSmirnov; si la hipótesis nula de normalidad se mantiene, entonces la media aritmética y la desviación estándar son los descriptores más adecuados de la distribución, pudiéndose transformar las puntuaciones brutas $(\mathrm{PB})$ en puntuaciones $\mathrm{T}(\mathrm{PT})$, definidas en una escala de intervalo de media 100 y desviación estándar 15, más sencilla de interpretar con base en los datos normativos (N=964): PT = 100+15 [(PB- Media aritmética)/Desviación estándar].

La capacidad discriminatoria de cada uno de los 27 elementos se estudió a través de una contrastación de medias. Se definieron dos grupos según la puntuación en la escala o suma de elementos (ST). Un grupo estuvo formado por el $27 \%$ inferior de la distribución de ST y otro por el $27 \%$ superior. Al existir una correlación positiva entre el elemento y el test, hay mayor probabilidad de hallar una diferencia significativa entre grupos cercanos a la media $(50 \%$ por encima de la media y $50 \%$ por abajo) que entre grupos más extremos ( $25 \%$ por arriba y $25 \%$ por abajo). No obstante, en la medida que se emplean grupos con puntajes más extremos en la escala aumenta el error estándar de la diferencia. Kelly ${ }^{10}$ muestra cómo la relación entre la magnitud de la diferencia y la del error estándar se optimiza si la computación de la diferencia se basa en el $27 \%$ de la distribución del test con los puntajes más altos y el 27\% más bajos. Se contrasta la diferencia de medias entre estos dos grupos en cada elemento empleando la prueba no paramétrica U de Mann-Whitney. Se considera que el elemento es discriminatorio, si se puede rechazar la hipótesis nula de la igualdad de media, con una probabilidad de error $<0.01$.
La confiabilidad o consistencia interna de los elementos se estudia por la correlación lineal entre dicho elemento y el resto de la escala, es decir la suma de los demás elementos excluido el elemento en cuestión. Se estima por la correlación biserial-puntual.

La consistencia interna de la escala se estudia por $\alpha$ de Cronbach. La estructura factorial se calcula por la técnica de análisis factorial, empleando como método de extracción de factores: el de componentes principales con una normalización de las variables tipo Kaiser. Se realiza una rotación ortogonal de la matriz factorial por el método varimax. Los factores se definen por las saturaciones de los elementos en la matriz factorial rotada.

La validez de constructo de SEA-27 con relación a otras variables se estudia por su correlación lineal de Pearson con las variables numéricas, por la diferencia de medias ( $t$ de Student para muestras independientes) con las dicotómicas y el ANOVA con las politómicas. Las variables numéricas empleadas fueron: las 10 referidas a la actitud hacia el uso del preservativo y planificación de las relaciones sexuales (D1 a D10), actitud hacia la monogamia, acuerdo respecto al reactivo "es muy difícil contraer el SIDA, aunque uno no se proteja", valoración de la información que se tiene sobre la sexualidad, edad de la primera relación sexual y número de personas con las que se ha tenido relaciones sexuales. Asimismo, se correlaciona con la edad y clase social. Las variables dicotómicas son: haber tenido o no relaciones sexuales, la utilización de preservativo en la primera relación sexual, la planificación de la primera relación sexual y empleo de preservativo en las relaciones sexuales actuales, donde se compara la diferencia en la media en ST entre dos grupos que definen cada una de estas cuatro variables por medio de la prueba $t$ de Student para grupos independientes. De forma previa se contrasta la igualdad de varianza por la F de Fisher-Snedecor. Las variables politómicas serían el estado civil, la clase social y la valoración de la eficacia del preservativo.

Finalmente, se aportan datos sobre la valoración de la peligrosidad de las distintas conductas de riesgo. Se define 15 variables: Riesgo A, a Riesgo O. Cada una puede tomar tres valores. Por ejemplo, Riesgo A toma el valor 1 si la opción A ("Tener relaciones sexuales sin condón") es elegida en primer lugar, 2 si es elegida en segundo lugar y 3 si es elegida en tercer lugar. Se realiza un análisis de varianza con cada una de las 15 variables. Sus tres valores definen los tres grupos de medidas independientes $(1,2$ y 3$)$ y aparece como variable dependiente ST; es decir, se contrastan las medias de ST en los tres grupos definidos por cada una 
de las 15 variables Riesgo A, a Riesgo O. Si el estadístico $\mathrm{F}$ permite rechazar la hipótesis nula de igualdad de medias con una $p<0.05$, entonces se calcula la diferencia mínima significativa entre dichas medias. Primero, se contrasta el supuesto de homosedasticidad por el Test de Levene de igualdad de varianzas erráticas. A continuación, si se puede mantener el supuesto de homosedasticidad, la diferencia mínima significativa se calcula por la prueba LSD; y si no se puede mantener dicho supuesto, por la prueba de Tamhane's $\mathrm{T} 2$.

\section{Resultados}

La suma de elementos de la escala ST tiene una media de 95.14 y una desviación estándar de 25.80. Media (95) y mediana (94) prácticamente coinciden. La moda es un valor algo inferior (82). Por la prueba de Kolmogorov-Smirnov $\left(\mathrm{Z}_{\mathrm{k}-\mathrm{s} \text { (Normal) }}=1.369 ; p=0.047\right)$, se puede mantener la hipótesis nula de normalidad con una $p>0.025$ (considerando una formulación bilateral para la hipótesis nula). Por tanto, a la distribución de ST se la puede considerar como una curva normal N (95.14; 25.8). Las puntuaciones de ST se pueden transformar en una escala de puntuaciones $\mathrm{T}$ (PT), es decir en una escala con una media de 100 y desviación estándar de 15, aplicando la fórmula: $\mathrm{PT}=100+15$ $((X-95.14) / 25.8)$, hallándose comprendido el $68 \%$ de la distribución entre 85 y 115, 96\% entre 70 y 130, y 99\% entre 55 y 145 .

La confiabilidad de ST, por la $\alpha$ de Cronbach, es alta con un valor de 0.89 , y muy próxima al esperado de 0.91 . Todos los elementos son discriminatorios y presentan una correlación significativa $(p<0.01)$ con el resto de la escala que oscila de 0.284 a 0.654 , como se puede ver en el cuadro I.

Aparece una estructura tetradimensional que explica $58.47 \%$ de la varianza total, tal como se puede ver en el cuadro II. Aunque aparecen cuatro factores, la estructura se ajusta bien a las tres subescalas propuestas. La tercera subescala es la que se desdobla en los factores STF2 y STF3.

- El primer factor (STF1) comprende los 11 elementos (A1 a A11) que estiman la capacidad para decir no a las relaciones sexuales bajo diferentes circunstancias. Explica 25.82\% de la varianza total y presenta una consistencia interna muy elevada, de 0.92 .

- El segundo factor (STF2) comprende los ocho elementos ( $\mathrm{C} 1$ a $\mathrm{C} 8$ ) referentes al uso de preservativo. Explica el 14.29\% de la varianza total.
Su consistencia interna es moderadamente alta de 0.75 .

- El tercer factor (STF3) comprende cuatro elementos (C9 a C12) sobre el matrimonio, la fidelidad y el hablar de las relaciones sexuales con los padres. Explica el 9.6\% de la varianza y tiene una consistencia interna moderadamente alta de 0.75 .

- El cuarto factor (STF4) está compuesto por los cuatro elementos (B1 a B4) que abordan sobre la capacidad para preguntar al compañero sobre las relaciones sexuales anteriores y otras conductas de riesgo como consumo de drogas. Explica el $8.76 \%$ de la varianza total y tiene una consistencia interna alta de 0.8 .

Se observa como tendencia que las conductas de riesgo consideradas más peligrosas por las personas autoeficaces son las relaciones sin preservativo, con prostitutas y homosexuales, y que en las situaciones donde no hay control aparece, un nivel medio más bajo de autoeficacia. No obstante, las diferencias de medias en ningún caso son significativas.

Todas las variables (D1 a D10) sobre uso del preservativo y planificación de las relaciones sexuales correlacionan significativamente con la suma de los elementos de la escala ST y especialmente con el tercero, referente al matrimonio, la fidelidad y el hablar de las relaciones sexuales con los padres, al igual que la actitud hacia la monogamia, y estar en desacuerdo con que es muy difícil contraer el SIDA, aunque uno no se proteja (cuadro III).

Los adolescentes que han tenido relaciones sexuales son el 30\%. En su primera relación sexual 32\% empleó el preservativo y $34 \%$ la planificó (cuadro IV). Tuvo lugar en $20 \%$ de los casos en la casa de los padres. La edad media de la primera relación en el varón fue de $14.86 \pm 2.84$ y en la mujer de $15.76 \pm 4.06$. La relación entre la edad de inicio de la primera relación sexual y la escala de autoeficacia (ST) es negativa y significativa $(\mathrm{r}=-0.170, p=0.000)$, al igual que con su primer (SEAF1), tercer (SEAF3) y cuarto factor (SEAF4). A mayor autoeficacia, mayor edad de inicio (cuadro III).

El número medio de compañeros sexuales es de 2 a 3. Existe una relación significativa entre número de compañeros sexuales y autoeficacia (ST, $\mathrm{r}=-0.169$, $p=0.000$ ), al igual que con su cuarto factor (SEAF4). A mayor autoeficacia, menor número de compañeros sexuales (cuadro III). El 39\% de los adolescentes sexualmente activos dice protegerse con preservativos en sus relaciones sexuales actuales frente a un $61 \%$ que no lo hace (cuadro IV). Las razones por las que 


\section{Cuadro I}

\section{INDICES DE DISCRIMINACIÓN, CONFIABILIDAD Y VALIDEZ DE LOS ELEMENTOS DE LA ESCALA DE AUTOEFICACIA para la prevención del SIDA-SEA-27. Monterrey, NueVo León, México, 1998}

\begin{tabular}{|c|c|c|c|c|c|}
\hline Elementos & M & DS & Discriminación & Fiabilidad & Validez \\
\hline A 1 & 3.70 & 1.61 & $U=2317 p<0.000$ & $r=0.56 p<0.000$ & $r=0.033 p=0.414$ \\
\hline A_2 & 3.66 & 1.66 & $U=1586 p<0.000$ & $r=0.618 p<0.000$ & $r=0.105 p=0.010$ \\
\hline $\bar{A} 3$ & 3.73 & 1.66 & $U=1660 p<0.000$ & $r=0.616 p<0.000$ & $r=0.076 p=0.062$ \\
\hline A_4 & 3.57 & 1.43 & $U=1773.5 p<0.000$ & $r=0.598 p<0.000$ & $r=0.036 p=0.376$ \\
\hline A_5 & 3.59 & 1.46 & $U=1819.5 p<0.000$ & $r=0.584 p<0.000$ & $r=0.047 p=0.250$ \\
\hline $\bar{A} 6$ & 3.32 & 1.60 & $U=2012 p<0.000$ & $r=0.569 p<0.000$ & $r=0.112 p=0.007$ \\
\hline$\overline{A \_7}$ & 3.55 & 1.53 & $U=2093.5 p<0.000$ & $r=0.554 p<0.000$ & $r=0.074 p=0.071$ \\
\hline$\overline{A \_8}$ & 3.56 & 1.74 & $U=1660 p<0.000$ & $r=0.611 p<0.000$ & $r=0.053 p=0.190$ \\
\hline A_9 & 3.44 & 1.72 & $U=1327.5 p<0.000$ & $r=0.654 p<0.000$ & $r=0.092 p=0.025$ \\
\hline A_10 & 3.62 & 2.92 & $U=1351 p<0.000$ & $r=0.426 p<0.000$ & $r=-0.022 p=0.604$ \\
\hline A_11 & 3.27 & 1.63 & $U=1935 p<0.000$ & $r=0.595 p<0.000$ & $r=0.086 p=0.038$ \\
\hline$B_{-} 1$ & 3.87 & 1.44 & $U=3765.5 p<0.000$ & $r=0.455 p<0.000$ & $r=0.049 p=0.231$ \\
\hline $\bar{B} \_2$ & 3.84 & 1.41 & $U=3163 p<0.000$ & $r=0.48 p<0.000$ & $r=0.102 p=0.012$ \\
\hline B_3 & 3.55 & 2.75 & $U=2705.5 p<0.000$ & $r=0.284 p<0.000$ & $r=0.099 p=0.015$ \\
\hline B_4 & 3.18 & 1.63 & $U=2914 p<0.000$ & $r=0.481 p<0.000$ & $r=0.138 p=0.001$ \\
\hline$C_{-} 1$ & 3.92 & 1.44 & $U=3973.5 p<0.000$ & $r=0.437 p<0.000$ & $r=0.125 p=0.002$ \\
\hline $\bar{c} 2$ & 3.78 & 1.39 & $U=3892.5 p<0.000$ & $r=0.414 p<0.000$ & $r=0.102 p=0.012$ \\
\hline $\mathrm{C}_{-} 3$ & 3.25 & 2.82 & $U=1581.5 p<0.000$ & $r=0.389 p<0.000$ & $r=0.165 p<0.000$ \\
\hline$C_{-} 4$ & 2.99 & 1.72 & $U=1698.5 p<0.000$ & $r=0.569 p<0.000$ & $r=0.145 p=0.001$ \\
\hline $\bar{C} 5$ & 3.64 & 1.57 & $U=1712.5 p<0.000$ & $r=0.603 p<0.000$ & $r=0.137 p=0.001$ \\
\hline $\mathrm{C}_{-} 6$ & 3.64 & 2.45 & $U=2119.5 p<0.000$ & $r=0.417 p<0.000$ & $r=0.189 p<0.000$ \\
\hline $\bar{C} 7$ & 3.40 & 1.53 & $U=2719.5 p<0.000$ & $r=0.515 p<0.000$ & $r=0.257 p<0.000$ \\
\hline $\bar{c} 8$ & 3.29 & 2.82 & $U=2116.5 p<0.000$ & $r=0.338 p<0.000$ & $r=0.176 p<0.000$ \\
\hline$C_{-} 9$ & 3.54 & 1.68 & $U=4011.5 p<0.000$ & $r=0.386 p<0.000$ & $r=0.171 p<0.000$ \\
\hline$C_{-} 10$ & 3.74 & 1.52 & $U=5096.5 p<0.000$ & $r=0.348 p<0.000$ & $r=0.210 p<0.000$ \\
\hline C_11 & 3.12 & 1.63 & $U=3869.5 p<0.000$ & $r=0.372 p<0.000$ & $r=0.166 p<0.000$ \\
\hline $\bar{c}$ & 3.35 & 1.66 & $U=4133 p<0.000$ & $r=0.337 p<0.000$ & $r=0.191 p<0.000$ \\
\hline
\end{tabular}

M Media DS Desviación estándar

no se emplea el preservativo son: por incomodidad o pérdida del placer $(40 \%)$ y económicas $(30 \%)$. El primer motivo esgrimido no era esperado en la población adolescente, cuando sí podría ser más factible en hombres maduros o ancianos.

La edad correlaciona significativamente sólo con el segundo factor de SEA-27 ( $r=0.117, p=0.01)$. A mayor edad, mayor autoeficacia percibida para usar el preservativo (SEAF2). La clase social sólo correlaciona con la escala SEA-27 $(\mathrm{r}=0.109, p=0.020)$ y su segundo factor $(r=0,110, p=0.003)$. A mayor clase social, mayor autoeficacia percibida para evitar conductas sexuales de riesgo (SEA-27) y para usar el preservativo (SEAF2) (cuadro III).
Los adolescentes que no han tenido relaciones sexuales puntúan significativamente más alto $(t=-5.41$, $p<0.000)$ en autoeficacia (ST) que los que sí tienen relaciones. También aquellos que planificaron su primera relación sexual puntúan significativamente más $(t=2.29, p=0.026)$ que los que no que planificaron. Aunque las personas que utilizaron el preservativo en su primera relación sexual tienden a puntuar más en autoeficacia (ST), la diferencia no es significativa ( $p=0.290)$. Lo mismo se observa respecto al empleo de preservativo en las relaciones actuales, donde puntúan más los que sí lo hacen, pero la diferencia de medias en ST no es significativa $(p=0.061)$ (cuadro V). La diferencia por estado civil no es significativa. Los 


\section{Matriz factorial rotada de la esCALA de aUtoeficacia SEA-27}

Items

SEAF1 SEAF2 SEAF3 SEAF4

( $Q$ ué tan seguro estás de ser capaz de decir no, cuando te propone tener relaciones sexuales ...

A1 ... alguien conocido hace 30 días o menos?

\begin{tabular}{llll}
0.807 & 0.02584 & 0.02347 & 0.09808 \\
\hline
\end{tabular}

A2 ... alguien cuya historia sexual es desconocida para ti?

$\begin{array}{llll}0.840 & 0.117 & -0.03102 & 0.08128\end{array}$

A3 ... alguien cuya historia de drogas es desconocida para ti?

$\begin{array}{llll}0.806 & 0.138 & -0.04767 & 0.128\end{array}$

A4 ... alguien a quien has tratado con anterioridad?

$\begin{array}{llll}0.794 & 0.09742 & 0.134 & 0.03042\end{array}$

A5 ... alguien a quien deseas tratar de nuevo?)

\begin{tabular}{llll}
0.773 & 0.06507 & 0.125 & 0.09346 \\
\hline
\end{tabular}

A6 ... alguien con quien ya has tenido relaciones sexuales?)

\begin{tabular}{llll}
0.708 & 0.153 & 0.09188 & 0.05689 \\
\hline
\end{tabular}

A 7 ... alguien a quien necesitas que se enamore de ti?

$\begin{array}{llll}0.759 & 0.07476 & 0.148 & -0.01886\end{array}$

A8 ... alguien que te presiona a tener relaciones sexuales?)

0.825

A 9 ... alguien con quien has estado bebiendo alcohol?)

A 10 ... alguien con quien has estado utilizando drogas?)

0.849

$\begin{array}{lll}0.08924 & -0.02879 & 0.129\end{array}$

A11 ... alguien y tu nivel de excitación sexual es muy alto?

$\begin{array}{llll}0.576 & 0.154 & -0.202 & 0.165 \\ 0.786 & 0.140 & 0.08762 & 0.005058\end{array}$

(¿Q ué tan seguro estás de ser capaz de...

B1 ... preguntar a tu novio/a si se ha inyectado alguna droga?)

$\begin{array}{llll}0.230 & 0.193 & -0.02011 & 0.725\end{array}$

B2 ... discutir sobre la prevención del SIDA con tu novio/a?)

$\begin{array}{llll}0.08720 & 0.323 & 0.181 & 0.703\end{array}$

B3 ... preguntar a tu novio/a sobre sus relaciones sexuales tenidas en el pasado?)

$\begin{array}{llll}0.05094 & 0.08153 & 0.08373 & 0.654\end{array}$

B4 ... preguntar a tu novio/a si ha tenido alguna experiencia homosexual?)

$\begin{array}{llll}0.142 & 0.197 & 0.195 & 0.733\end{array}$

(¿Q ué tan seguro estás de ser capaz de ...

C1 ... usar el condón cada vez que tengas relaciones sexuales?

C2 ... usar correctamente el condón?

C 3 ... usar el condón durante el acto sexual después de que has estado bebiendo alcohol?)

$\begin{array}{llll}-0.02103 & 0.778 & 0.173 & 0.102\end{array}$

$\begin{array}{rrrr}-0.06772 & 0.770 & 0.174 & 0.124\end{array}$

C 4 ... usar el condón durante el acto sexual después de que has estado consumiendo

o utilizado alguna droga? (mariguana, resistol, tinner))

$\begin{array}{llll}0.187 & 0.531 & -0.01720 & 0.08012\end{array}$

\begin{tabular}{|c|c|c|c|c|}
\hline o utilizado alguna droga? (mariguana, resistol, tinner)) & 0.282 & 0.642 & 0.006607 & 0.173 \\
\hline C5 ... insistir en el uso del condón durante el acto sexual incluso si tu novio/a prefiere no usarlo?) & 0.203 & 0.697 & 0.241 & 0.185 \\
\hline C6 ... negarte a tener relaciones sexuales si tu pareja no acepta usar el condón?) & 0.195 & 0.484 & 0.237 & 0.002580 \\
\hline C7 ... contar siempre con el dinero suficiente para comprar condones?) & 0.08864 & 0.728 & 0.180 & 0.142 \\
\hline C8 ... acudir a la tienda a comprar condones?) & 0.08035 & 0.457 & 0.101 & 0.150 \\
\hline C9 ... no tener relaciones sexuales hasta contraer matrimonio?) & 0.102 & 0.210 & 0.803 & 0.03195 \\
\hline C10 ... mantener relaciones sexuales con una sola persona para toda la vida?) & 0.03892 & 0.245 & 0.794 & -0.01055 \\
\hline C11 ... platicar con tu papá sobre temas sexuales?) & 0.009275 & 0.232 & 0.598 & 0.304 \\
\hline C12 ... platicar con tu mamá sobre temas sexuales?) & 0.01066 & 0.148 & 0.739 & 0.211 \\
\hline$\lambda$ (autovalor) & 6.971 & 3.859 & 2.592 & 2.365 \\
\hline \% de varianza total explicada & 25.820 & 14.294 & 9.6 & 8.759 \\
\hline$\alpha$ de Cronbach (consistencia interna) & 0.9204 & 0.7481 & 0.756 & 0.8 \\
\hline
\end{tabular}

casados presentan la media más alta en ST y los separados/divorciados la más baja, sin que la diferencia llegue a ser significativa $(\mathrm{F}=1.14, p=0.326)$. Igualmente, por clase social la media más alta aparece en la clase alta/media-alta y la inferior en la baja, sin ser significativa la diferencia $(\mathrm{F}=2.803, p=0.062$,) (cuadro $\mathrm{V}$ ).

$\mathrm{Al}$ estudiar una a una la valoración de las conductas de riesgo no se halla diferencia significativa $(p \leq 0.05)$ en la media de puntuación en ST en ninguna opción de orden. Si se observa una tendencia a ser mayor la media en ST, como primera opción, en riesgo C (la infidelidad con la pareja); como segunda opción, en riesgo $\mathrm{G}$ (tener relaciones sexuales con alguien que está drogado); y como tercera opción, en riesgo $\mathrm{O}$ (tener relaciones sexuales con desconocidos/as) (cuadro V). 


\section{Cuadro III \\ Validez de constructo de la escala de autoeficacia para la preVención del SIDA-SEA-27 y sus cuatro factores: validez convergente-divergente. Monterrey, Nuevo León, México 1998}

\begin{tabular}{|c|c|c|c|c|c|}
\hline Elementos & ST & SEAF1 & SEAF2 & SEAF3 & SEAF4 \\
\hline D1 (La mujer es la que debe solicitar el uso del condón) & $r=0.130 p<0.004$ & $r=-0.049 p=0.215$ & $r=0.124 p<0.000$ & $r=0.135 p<0.000$ & $r=0.099 p=0.005$ \\
\hline D2 (El hombre es el que debe comprar los condones) & $r=0.107 p=0.017$ & $r=0.012 p=0.766$ & $r=0.041 p=0.245$ & $r=0.165 p<0.000$ & $r=0.083 p=0.017$ \\
\hline \multicolumn{6}{|l|}{ D3 (El hombre es el responsable de la protección durante } \\
\hline la relación sexual) & $r=0.03 p=0.508$ & $r=-0.08 p=0.043$ & $r=-0.011 p=0.749$ & $r=0.065 p=0.095$ & $r=0.146 p<0.000$ \\
\hline D4 (La mujer debe de traer los condones) & $r=0.157 p<0.000$ & $r=0.077 p=0.050$ & $r=0.077 p=0.029$ & $r=0.116 p=0.003$ & $r=0.068 p=0.053$ \\
\hline D5 (Las relaciones sexuales deben ser espontáneas) & $r=-0.035 p=0.438$ & $r=0.038 p=0.340$ & $r=0.015 p=0.677$ & $r=-0.041 p=0.299$ & $r=-0.117 p=0.001$ \\
\hline D6 (El uso del condón es bueno para mi salud) & $r=0.118 p=0.009$ & $r=0.029 p=0.465$ & $r=0.136 p<0.000$ & $r=0.282 p<0.000$ & $r=0.045 p=0.198$ \\
\hline D7 (El uso del condón disminuye la satisfacción sexual) & $r=0.040 p=0.384$ & $r=0.123 p=0.002$ & $r=-0.027 p=0.467$ & $r=0.008 p=0.850$ & $r=-0.052 p=0.154$ \\
\hline D8 (Mi situación económica me permite comprar condones) & $r=0.211 p<0.000$ & $r=0.076 p=0.061$ & $r=0.184 p<0.000$ & $r=0.286 p<0.000$ & $r=0.077 p=0.034$ \\
\hline \multicolumn{6}{|l|}{ D9 (Las relaciones sexuales se disfrutan más si se planifican } \\
\hline con tiempo) & $r=0.241 p<0.000$ & $r=0.077 p=0.054$ & $r=0.175 p<0.000$ & $r=0.185 p<0.000$ & $r=0.317 p<0.000$ \\
\hline \multicolumn{6}{|l|}{ Mono (Las relaciones sexuales sólo deben tenerse con } \\
\hline la misma persona toda la vida) & $r=0.219 p<0.000$ & $r=0.14 p<0.000$ & $r=0.121 p=0.001$ & $r=0.093 p=0.016$ & $r=0.445 p<0.000$ \\
\hline \multicolumn{6}{|l|}{ D10 (Prefiero arriesgarme a contraer una enfermedad, } \\
\hline PSIDA (EI SIDA es muy difícil que me dé, aunque no me proteja) & $r=-0.083 p=0.067$ & $r=-0.098 p=0.014$ & $r=-0.093 p=0.008$ & $r=-0.081 p=0.039$ & $r=0.016 p=0.640$ \\
\hline Sinfo (Consideras que la información que tienes sobre el SIDA es) & $r=0.100 p=0.031$ & $r=-0.030 p=0.465$ & $r=0.121 p=0.001$ & $r=0.125 p=0.002$ & $r=0.207 p=0.000$ \\
\hline Edad & $r=0.042 p=0.351$ & $r=0.038 p=0.329$ & $r=0.117 p=0.010$ & $r=0.033 p=0.396$ & $r=-0.042 p=0.225$ \\
\hline Clase social & $r=0.109 p=0.020$ & $r=0.055 p=0.185$ & $r=0.110 p=0.003$ & $r=0.047 p=0.245$ & $r=0.061 p=0.095$ \\
\hline Edad de la primera relación sexual & $r=-0.170 p=0.000$ & $r=-0.071 p=0.068$ & $r=-0.010 p=0.763$ & $r=-0.123 p=0.001$ & $r=-0.213 p=0.000$ \\
\hline úmero de parejas sexuales & $r=-0.169 p=0.000$ & $r=-0.074 p=0.058$ & $r=-0.007 p=0.848$ & $r=-0.030 p=0.431$ & $r=-0.202 p=0.000$ \\
\hline
\end{tabular}

 de aUtoeficacia para la PReVención del SIDA, SEgún su aCtividad seXual. Monterrey, Nuevo León, México 1998}

\begin{tabular}{|c|c|c|c|c|c|c|c|c|c|c|c|c|}
\hline & & relsexua & & & Condón & & & rsexfu & & & conact & \\
\hline & $n$ & $\% 964$ & \%* 894 & $n$ & $\% 271$ & \%*211 & $n$ & $\% 271$ & $\% * 252$ & $n$ & $\% 271$ & $\% * 230$ \\
\hline Sí & 271 & 28 & 30 & 68 & 25 & 32 & 87 & 32 & 34 & 90 & 33 & 39 \\
\hline No & 623 & 65 & 70 & 143 & 53 & 68 & 165 & 61 & 66 & 140 & 52 & 61 \\
\hline s.d. & 70 & 7 & & 60 & 22 & & 19 & 7 & & 41 & 15 & \\
\hline Tot & 964 & 100 & 100 & 271 & 100 & 100 & 271 & 100 & 100 & 271 & 100 & 100 \\
\hline
\end{tabular}

\% Porcentaje calculado incluyendo casos sin datos (s.d.) en la variable

\%* Porcentaje calculado excluyendo los casos sin datos (s.d.) en la variable

Respecto a la valoración de la eficacia del condón, la escala SEA-27 no discrimina $(p=0.083)$ significativamente entre los tres niveles propuestos: (a) de 95 a $100 \%$; (b) de 90 a 100\% y (c) menos de $90 \%$. Aunque sí se observa una tendencia a valorar más eficaces los preservativos cuanto mayor es la media en la escala SEA-27 (cuadro V).
Las demandas de información más frecuentes en la muestra se refieren al SIDA (50\%), infecciones de transmisión sexual $(20 \%)$, embarazo $(10 \%)$, abortos (5\%) y métodos anticonceptivos (3\%). Las fuentes de información más importantes son la escuela (29\%) y los amigos, y la mamá (12\%). El papá sólo lo es en 5\% de los casos. 


\section{Cuadro V \\ Validez de constructo de la escala de autoeficacia para la prevención del SiDA-SEA-27; contrastación de medias. Monterrey, Nuevo León, México 1998}

\begin{tabular}{|c|c|c|c|c|c|c|c|c|c|c|c|}
\hline \multirow[b]{3}{*}{ Variables } & \multicolumn{6}{|c|}{ Opciones de las variables } & \multicolumn{5}{|c|}{ t de Student } \\
\hline & \multicolumn{3}{|c|}{ Sí } & \multicolumn{3}{|c|}{ No } & \multicolumn{2}{|c|}{ Igual de varianzas en ST } & \multicolumn{3}{|c|}{ Igual de medias en ST } \\
\hline & $\mathrm{n}$ & M & DS & $n$ & M & DS & $F$ & $p$ & t & g.l & $p$ \\
\hline Condón & 32 & 91.09 & 22.02 & 75 & 85.68 & 24.91 & 0.17 & 0.68 & 1.064 & 105 & 0.290 \\
\hline Rsexfue & 40 & 94.22 & 29.58 & 89 & 82.54 & 18.92 & 10.11 & 0.002 & 2.29 & 53.84 & 0.026 \\
\hline Conact & 37 & 92.86 & 24.06 & 86 & 84.50 & 21.76 & 1.04 & 0.31 & 1.893 & 121 & 0.061 \\
\hline Relsexual & 134 & 85.81 & 22.98 & 350 & 98.81 & 25.37 & 7.56 & 0.006 & -5.41 & 264.2 & 0.000 \\
\hline Riesgo & \multicolumn{3}{|c|}{1} & \multicolumn{3}{|c|}{2} & \multicolumn{3}{|c|}{3} & \multicolumn{2}{|c|}{ ANOVA } \\
\hline SEA-27 & $\mathrm{n}$ & M & DS & $\mathrm{n}$ & M & DS & $\mathrm{n}$ & M & DS & $\mathrm{F}$ & $p$ \\
\hline RiesgoA & 43 & 92.4 & 22.3 & 44 & 100.6 & 26.6 & 150 & 95.1 & 24.5 & 1.29 & 0.275 \\
\hline RiesgoB & 44 & 96.8 & 24.3 & 70 & 98.1 & 23.2 & 53 & 95.7 & 26.9 & .149 & 0.861 \\
\hline RiesgoC & 18 & 101.1 & 27.6 & 26 & 89.4 & 19.6 & 18 & 80.0 & 31.1 & 3.02 & 0.056 \\
\hline RiesgoD & 12 & 92.3 & 22.5 & 13 & 77.4 & 27.0 & 8 & 84.9 & 21.9 & 1.18 & 0.321 \\
\hline RiesgoE & 13 & 89.5 & 25.0 & - & - & - & 6 & 95.5 & 29.7 & .214 & 0.650 \\
\hline Riesgof & 10 & 69.6 & 30.3 & 13 & 94.9 & 27.2 & 2 & 64.0 & 31.1 & 2.65 & 0.093 \\
\hline RiesgoG & 25 & 84.5 & 17.6 & 28 & 94.8 & 24.8 & 12 & 93.3 & 31.3 & 1.3 & 0.270 \\
\hline RiesgoH & 51 & 98.9 & 23.5 & 34 & 94.6 & 27.9 & 32 & 106.3 & 20.9 & 1.96 & 0.146 \\
\hline Riesgol & 16 & 93.0 & 23.2 & 14 & 84.6 & 21.4 & 10 & 92.7 & 22.3 & .629 & 0.539 \\
\hline Riesgol & 10 & 88.7 & 39.3 & 5 & 96.0 & 21.7 & 7 & 84.6 & 28.6 & .176 & 0.840 \\
\hline RiesgoK & 62 & 93.8 & 24.6 & 72 & 98.2 & 25.2 & 54 & 96.8 & 26.6 & .520 & 0.596 \\
\hline RiesgoL & 56 & 101.6 & 28.9 & 66 & 93.3 & 24.3 & 83 & 95.6 & 24.5 & 1.68 & 0.189 \\
\hline$\overline{\text { RiesgoM }}$ & 3 & 110.3 & 24.1 & 4 & 102.2 & 34.6 & 1 & 96.0 & - & .103 & 0.904 \\
\hline RiesgoN & 7 & 100.3 & 28.2 & 10 & 92.8 & 28.6 & 5 & 62.8 & 15.5 & 3.24 & 0.062 \\
\hline Riesgo 0 & 92 & 93.0 & 25.9 & 53 & 85.5 & 23.1 & 33 & 96.2 & 24.9 & 2.27 & 0.106 \\
\hline Efcondón & \multicolumn{3}{|c|}{95 a $100 \%$} & \multicolumn{3}{|c|}{90 al $100 \%$} & \multicolumn{3}{|c|}{ menos del $90 \%$} & \multicolumn{2}{|c|}{ AN OVA } \\
\hline \multirow[t]{2}{*}{ ST } & $\mathrm{n}$ & M & DS & $\mathrm{n}$ & M & DS & $\mathrm{n}$ & M & DS & $\mathrm{F}$ & $P$ \\
\hline & 128 & 99.1 & 25.8 & 166 & 94.0 & 23.0 & 123 & 92.4 & 27.5 & 2.50 & 0.083 \\
\hline Est. Civil & \multicolumn{3}{|c|}{ Soltero } & \multicolumn{3}{|c|}{ Casado } & \multicolumn{3}{|c|}{ Separado / divorci. } & \multicolumn{2}{|c|}{ ANOVA } \\
\hline \multirow[t]{2}{*}{ ST } & $n$ & M & DS & $n$ & M & DS & $n$ & M & DS & $\mathrm{F}$ & $P$ \\
\hline & 30 & 90.2 & 21.7 & 28 & 95.2 & 30.5 & 12 & 81.7 & 23.8 & 1.14 & 0.326 \\
\hline Clase Soc. & \multicolumn{3}{|c|}{ Alta / Media-alta } & \multicolumn{3}{|c|}{ Media-baja } & \multicolumn{3}{|c|}{ Baja } & \multicolumn{2}{|c|}{ ANOVA } \\
\hline \multirow[t]{2}{*}{ ST } & $n$ & M & DS & $n$ & M & DS & $n$ & M & DS & $\mathrm{F}$ & $P$ \\
\hline & 7 & 99.7 & 23.1 & 179 & 97.9 & 25.7 & 267 & 92.2 & 26.3 & 2.80 & 0.062 \\
\hline 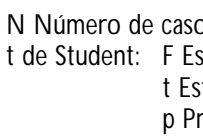 & $\begin{array}{l}\text { S } \\
\text { tadístic } \\
\text { sadístic } \\
\text { obabilic }\end{array}$ & $\begin{array}{l}\text { M } \\
\text { e contra } \\
\text { e contras } \\
\text { asociada }\end{array}$ & $\begin{array}{l}\text { ia de ST } \\
\text { on de igu } \\
\text { in de la is } \\
\text { alor t }\end{array}$ & $\begin{array}{l}\text { varianza } \\
\text { e medias }\end{array}$ & & $\begin{array}{l}\text { ación es } \\
\text { obabilid } \\
\text { rados d }\end{array}$ & $\begin{array}{l}\text { andar de S } \\
\text { lasociada } \\
\text { libertad }\end{array}$ & lor de F & & & \\
\hline ANOVA: & tadístic & e contra & e igualda & nedias & & nificat & de $F$ & & & & \\
\hline
\end{tabular}

\section{Conclusiones}

Se concluye que SEA-27 es una buena escala para medir autoeficacia sexual para prevenir el SIDA. Tiene buenas cualidades psicométricas de confiabilidad y validez. Su distribución se ajusta a la curva normal N(95.14, 25.80), cuyos estadísticos descriptivos se calcularon en una muestra no probabílistica $(\mathrm{n}=964)$ de tipo incidental (consultas de medicina general) de adolescentes (de 12 a 18 años) mexicanos neoleoneses, de los cuales ninguno tenía diagnóstico de SIDA ni VIH +.

Con base en estos datos tenemos 30\% de la población adolescente sexualmente activa, de los cuales sólo 39\% usa los preservativos, con 2 a 3 compañeros en sus primeros años de experiencia sexual. Teniendo en cuenta que la principal fuente de información es la 
escuela y hay un interés por saber sobre el SIDA, infecciones de transmisión sexual y embarazo, aparece como muy pertinente un programa psicodidáctico centrado en la escuela. Tras su aplicación, el giro de la incidencia creciente nos podrá dar la razón.

\section{Referencias}

1. 0 N UAID S.The G lobal strategy framework on HIV/SIDA. Ginebra, Suiza: O N UAIDS, 2001.

2. Amigos contra el SIDA. Estadísticas en México. Sistema de vigilancia epidemiológica del SIDA. [En red]. D isponible en:http://www.aids-sida.org/ estadist01.html\#tope. Mayo, 2001.

3. Bandura A. Self-efficacy mechanism in psychobiologic functioning. En: Schwarzer R ed, Self-efficay:Thought control of action. W ashington, DC: Hemisphere 1992;355-394.
4. Bandura A. Self-efficacy:Toward a unifying theory of behavioral change. Psychol Review 1977;84:191-215.

5. Kasen S,Vaugahan RD,W alter. Self-Efficacy for A ID S preventive behaviors among tenth grade students. Health Educ Q uaterly HJ 1992;19:187-202. 6. Bayés $R$,Villamarín $F, O$ choa 0 . El SIDA en los adolescentes mexicanos: Un análisis de las conductas de riesgo desde la teoría de la autoeficacia. Rev Psicológica Contemporánea 1995;5(2):46-55.

7. López F, PadillaV. SIDA, comunicación y educación; evaluación diagnóstica para el diseño, planeación y realización de un programa psicodidáctico del SIDA en jóvenes de 13 a 18 años del estado de $N$ uevo León. En: Ribeiro M, López RE. eds. Políticas sociales sectoriales: Tendencias actuales. Monterrey (N L): Editorial Universidad Autónoma de N uevo León, 1999;: :225-240.

8. López F, Salinas MC, Landero R. Se perciben más autoeficaces las mujeres en la prevención de conductas de riesgo sexual. Perspectivas Sociales 1999;2(2):77-86.

9. López F, PadillaV. Conductas de riesgo sexual y clase social: autoeficacia percibida. Rev Int Ciencias So ciales y H umanidades Sociotam .2000;10(2). 10. Kelly TL The selection of upper and lower groups for the validation of test items. J Educ Psychol 1939;30:17-24. 


\section{A péndice}

\section{Encuesta aplicada en el estudio para la validación de UNA escala de aUtoeficacia para preVeniR el SIDA}

Edad Sexo: Masc Fem

Estado Civil

Viven en tu casa otros parientes o hermanos(as) casados(as)

Ultimo grado escolar que terminado

¿Estudias actualmente? Sí No ¿Trabajas actualmente? Sí No

Col. en que vives:

\section{Escala de Autoeficacia para prevenir el SidA, de 27 elementos, SeA-27}

A continuación se presentan una serie de preguntas en las cuales vas a circular el grado de seguridad que creas tener
Nada Algo Medio Muy Total seguro seguro seguro seguro seguro

A) ¿Q ué tan seguro estás de ser capaz de decir $\mathrm{N}$ o, cuando te propone tener relaciones sexuales ...?

1. Alguien conocido hace 30 días o menos

2. Alguien cuya historia sexual es desconocida para ti

3. Alguien cuya historia de drogas es desconocida para ti?

4. Alguien a quien has tratado con anterioridad?

5. Alguien a quien deseas tratar de nuevo?

6. Alguien con quien ya has tenido relaciones sexuales

7. Alguien a quien necesitas que se enamore de ti

8. Alguien que te presiona a tener relaciones sexuales

9. Alguien con quien has estado bebiendo alcohol

10. Alguien con quien has estado utilizando drogas

11. Alguien y tu nivel de excitación sexual es muy alto

\begin{tabular}{rrrrr}
1 & 1 & 1 & 1 & 1 \\
\hline 2 & 2 & 2 & 2 & 2 \\
3 & 3 & 3 & 3 & 3 \\
4 & 4 & 4 & 4 & 4 \\
5 & 5 & 5 & 5 & 5 \\
6 & 6 & 6 & 6 & 6 \\
7 & 7 & 7 & 7 & 7 \\
8 & 8 & 8 & 8 & 8 \\
9 & 9 & 9 & 9 & 9 \\
10 & 10 & 10 & 10 & 10 \\
11 & 11 & 11 & 11 & 11
\end{tabular}

B) ¿Q ué tan seguro estás de ser capaz de

1. Preguntar a tu novio/a si se ha inyectado alguna droga?

2. Discutir sobre la prevención del SIDA con tu novio/a

3. Preguntar a tu novio/a sobre sus relaciones sexuales tenidas en el pasado?

4. Preguntar a tu novio/a si ha tenido alguna experiencia homosexual?

$\begin{array}{lllll}1 & 1 & 1 & 1 & 1 \\ 2 & 2 & 2 & 2 & 2 \\ 3 & 3 & 3 & 3 & 3 \\ 4 & 4 & 4 & 4 & 4\end{array}$

C) ¿Q ué tan seguro estás de ser capaz de....

1. Usar el condón cada vez que tengas relaciones sexuales?

2. Usar correctamente el condón

3. Usar el condón durante el acto sexual después de que has estado bebiendo alcohol?

4. Usar el condón durante el acto sexual después de que has estado consumiendo 0 utilizado alguna droga? (mariguana, resistol, tinner)

5. Insistir en el uso del condón durante el acto sexual incluso si tu novio/a prefiere no usarlo?

6. Negarte a tener relaciones sexuales si tu pareja no acepta usar el condón?

7. Contar siempre con el dinero suficiente para comprar condones?

8. Acudir a la tienda a comprar condones?

9. No tener relaciones sexuales hasta contraer matrimonio?

$\begin{array}{lllll}1 & 1 & 1 & 1 & 1 \\ 2 & 2 & 2 & 2 & 2 \\ 3 & 3 & 3 & 3 & 3\end{array}$

10. Mantener relaciones sexuales con una sola persona para toda la vida?

11. Platicar con tu papá sobre temas sexuales

12. Platicar con tu mamá sobre temas sexuales

\begin{tabular}{rrrrr}
4 & 4 & 4 & 4 & 4 \\
\hline 5 & 5 & 5 & 5 & 5 \\
6 & 6 & 6 & 6 & 6 \\
7 & 7 & 7 & 7 & 7 \\
\hline 8 & 8 & 8 & 8 & 8 \\
9 & 9 & 9 & 9 & 9 \\
10 & 10 & 10 & 10 & 10 \\
11 & 11 & 11 & 11 & 11 \\
\hline 12 & 12 & 12 & 12 & 12
\end{tabular}

D) Expresa el grado de acuerdo que tengas

1. La mujer es la que debe de solicitar el uso del condón.

2. El hombre es el que debe de comprar los condones.

3. El hombre es el responsable de la protección durante la relación sexual.

4. La mujer debe de traer los condones.

5. Las relaciones sexuales deben de ser espontáneas.

6. El uso del condón es bueno para mi salud.

7. El uso del condón disminuye la satisfacción sexual.

8. Mi situación económica me permite comprar condones.

9. Las relaciones sexuales se disfrutan más si se planifican con tiempo.

10. Las relaciones sexuales solo deben de tenerse con la misma persona de por vida.

11. Prefiero arriesgarme a contraer una enfermedad, a no tener relaciones sexuales.

12. El SIDA es algo muy difícil que me de, aunque no me proteja.

13. Consideras que la información que tienes sobre sexualidad es

\begin{tabular}{rrrrr}
1 & 1 & 1 & 1 & 1 \\
2 & 2 & 2 & 2 & 2 \\
3 & 3 & 3 & 3 & 3 \\
4 & 4 & 4 & 4 & 4 \\
5 & 5 & 5 & 5 & 5 \\
6 & 6 & 6 & 6 & 6 \\
7 & 7 & 7 & 7 & 7 \\
\hline 8 & 8 & 8 & 8 & 8 \\
9 & 9 & 9 & 9 & 9 \\
10 & 10 & 10 & 10 & 10 \\
11 & 11 & 11 & 11 & 11 \\
\hline 12 & 12 & 12 & 12 & 12 \\
13 & 13 & 13 & 13 & 13
\end{tabular}




\section{Preguntas adicionales}

La información sobre sexualidad que tienes la obtuviste principalmente de: (Marca sólo aquella que consideres la más importante:
a) Amigos
b) Libros
c) Revistas
d) Periódicos
e) Televisión
f) Escuela
g) Papá

h) Mamá

Sobre qué tema de sexualidad te gustaría tener más información:

Q uién te ha proporcionado la información sobre sexualidad que consideras más importante:

Has tenido relaciones sexuales $\quad \mathrm{No} \quad \mathrm{Si}$

\section{¿CONTESTA LAS SIGUIEN TES PREGUNTAS SO LO SI HASTEN ID O RELACIO N ES SEXUALES!}

¿Utilizaste el condón en tu primera relación sexual? N N $\quad \mathrm{Si}$

¿A qué edad fue tu primera relación sexual? ——años

Tu primera relación sexual fue: Algo que planeaste Algo no planeado

En qué lugar fue tu primera relación sexual:

$\begin{array}{llll}\text { a) En una casa. } & \text { b) En un auto } & \text { c) En un hotel } & \text { d) En despoblado }\end{array}$

$\mathrm{N}$ úmero de personas con las que has tenido relaciones sexuales:

¿Utilizas condones en tus relaciones actuales? No Si

Menciona la razón principal por la cual no se usó el condón en alguna de tus relaciones sexuales ( 0 en todas)

¿C uál crees que es la efectividad del condón cuando se utiliza correcta y adecuadamente?

$\begin{array}{lll}\text { a) } 95 \text { al } 100 \% & \text { b) } 90 \text { al } 100 \% & \text { c) menos de } 90 \%\end{array}$

De las siguientes opciones, selecciona en base a su importancia las tres que consideres de más riesgo para contraer el SIDA.

Escribe en el paréntesis ( ) la letra que corresponda a tu respuesta.

a) Tener relaciones sexuales sin condón.

b) Recibir transfusiones de sangre

c) Ser infiel a la pareja

d) Tener relaciones sexuales anales

e) Tener relaciones sexuales por presión (amigos/as)

f) Combinar el alcohol y las relaciones sexuales

g) Tener relaciones sexuales con alguien estando drogado

h) Tener más de un(a) compañero(a) sexual

i) Tener relaciones sexuales ocasionales (no planificadas)

j) Tener relaciones sexuales con otra persona basándote en su apariencia saludable

k) Tener relaciones homosexuales

I) Tener relaciones sexuales con prostitutas

m) Tener relaciones sexuales con el novio o novia

n) Tener relaciones sexuales por placer

o) Tener relaciones sexuales con desconocidos o desconocidas

\section{RESPUESTAS}

() La de primer lugar en riesgo. () La de segundo lugar en riesgo. () La de tercer lugar en riesgo. 